\title{
Mechanism research on the interaction regulation of Escherichia and IFN- $\gamma$ for the occurrence of T2DM
}

\author{
Meijun Lv ${ }^{1,2 \#}$, Lisha Li ${ }^{1 \#} \wedge$, Weidong $\mathrm{Li}^{1,2}$, Fujie Yang ${ }^{1,2}$, Qiongying Hu ${ }^{1,2} \wedge$, Daqian Xiong ${ }^{1,2} \wedge$ \\ ${ }^{1}$ Department of Laboratory Medicine, Affiliated Hospital of Chengdu University of Traditional Chinese Medicine, Chengdu, China; ${ }^{2}$ School of \\ Medical Technology, Chengdu University of Traditional Chinese Medicine, Chengdu, China \\ Contributions: (I) Conception and design: M Lv; (II) Administrative support: L Li; (III) Provision of study materials or patients: Q Hu; (IV) Collection \\ and assembly of data: D Xiong; (V) Data analysis and interpretation: D Xiong; (VI) Manuscript writing: All authors; (VII) Final approval of \\ manuscript: All authors. \\ \#These authors contributed equally to this work. \\ Correspondence to: Daqian Xiong. Department of Laboratory Medicine, Affiliated Hospital of Chengdu University of Traditional Chinese Medicine/ \\ School of Medical Technology, Chengdu University of Traditional Chinese Medicine, Chengdu, China. Email: 705006714@qq.com.
}

Background: Type 2 diabetes mellitus (T2DM) is a major social and public health problem which may be induced by intestinal flora imbalance through inflammatory response, and the specific mechanism remains unclear. In this study, we aim to explore the interaction network of intestinal flora and cell inflammation in T2DM.

Methods: This a case-control study. Patients with T2DM was the case group and healthy people as control. The differences of cytokine expression levels between patients with T2DM and healthy controls were assessed by using flow cytometry. The diversity and abundance of intestinal flora were evaluated by using $16 \mathrm{~S}$ rRNA three-generation full-length sequencing technology.

Results: A total of 29 patients with T2DM and 28 healthy controls were included for analysis. Compared with the healthy control group, the expression levels of plasma cytokine interleukin-2 (IL-2) ( $\mathrm{P}=0.0000006)$, IL-6 $(\mathrm{P}=0.000193)$, tumor necrosis factor $\alpha(\mathrm{TNF}-\alpha)(\mathrm{P}=0.016)$, interferon- $\gamma(\mathrm{IFN}-\gamma)(\mathrm{P}=0.000036)$ and interleukin-17 (IL-17) ( $\mathrm{P}=0.004)$ were significantly up-regulated in T2DM patients, and the abundance of Megamonas_funiformis $(\mathrm{P}=0.0016)$ and Escherichia $(\mathrm{P}=0.049)$ in the intestine were significantly increased. In contrast, the abundance of Bacteroides_stercoris $(\mathrm{P}=0.0068)$, Bacteroides_uniformis $(\mathrm{P}=0.033)$, and Phascolarctobacterium_faecium $(\mathrm{P}=0.033)$ were decreased in T2DM patients. Further, differentially expressed Escherichia had a positive correlation with IFN- $\gamma(\mathrm{r}=0.73)$ by Pearson correlation analysis.

Conclusions: The interaction network between the intestinal bacteria Escherichia and the cytokine IFN- $\gamma$ may drive inflammation in visceral adipose tissue (VAT), indicating insulin signal transduction can be inhibited in adipocytes to induce insulin resistance.

Keywords: Type 2 diabetes mellitus (T2DM); intestinal flora; cytokines; insulin resistancet

Submitted Jun 28, 2021. Accepted for publication Sep 28, 2021.

doi: 10.21037/apm-21-2318

View this article at: https://dx.doi.org/10.21037/apm-21-2318

\footnotetext{
^ ORCID: Meijun Lv, 0000-0001-8521-3969; Lisha Li, 0000-0003-1736-6386; Weidong Li, 0000-0002-3316-8534; Qiongying Hu, 00000002-1718-4542; Daqian Xiong, 0000-0003-0692-563X.
} 


\section{Introduction}

Type 2 diabetes mellitus (T2DM) has gradually become a prominent social health problem worldwide with increasing incidence yearly. There were approximately 116 million diabetic patients in China in 2019 (1). T2DM is a metabolic disease characterized by hyperglycemia and caused by relatively insufficient insulin secretion or insulin resistance (2). However, the pathogenesis of T2DM is complicated, including heredity, lifestyle, environmental, as well as unknown factors (3). Some studies have indicated that intestinal flora disorders can mediate the occurrence of T2DM, particularly those inducing insulin resistance by regulating visceral adipose tissue (VAT) inflammation. However, the specific pathogenic mechanism of the disease remains unclear.

Numerous studies have demonstrated that chronic lowgrade inflammation caused by visceral fat accumulation is one factor that may induce insulin resistance. Adipose tissue macrophage (ATM) is a key mediator of VAT inflammation, and its secreted pro-inflammatory cytokines can cause the inflammatory infiltration of VAT. This process will cause ATM stimulate further and release more pro-inflammatory cytokines, which could affect the sensitivity of insulin target tissues through multiple mechanisms to increase the risk of insulin resistance (4). However, the activation of ATM depends on the stimulation of interferon- $\gamma(\operatorname{IFN}-\gamma)(5)$, a pro-inflammatory cytokine secreted by natural killer $(\mathrm{NK})$ cells. IFN- $\gamma$ can stimulate the differentiation of macrophages into pro-inflammatory M1 macrophages (6) and mediate the occurrence of VAT inflammation in vitro experiments, while obesity-related inflammation and the development of insulin resistance was reduced by IFN- $\gamma$ gene knockout in mice (7). A previous study has investigated the impact of low IFN- $\gamma$ and interleukin-10 (IL-10) levels on tumor necrosis factor- $\alpha$ (TNF- $\alpha$ ) and interleukin-6 (IL-6) production by $\mathrm{PHA}$-induced peripheral blood mononuclear cells in T2DM, in which found that IFN- $\gamma$ concentration in the T2DM group was significantly lower than that in the control group (T2DM 7,700.86 $\pm 3,037.77$ vs. control $10,672.69 \pm 5,625.50 \mathrm{pg} / \mathrm{mL} ; \mathrm{P}=0.048)$. However, TNF- $\alpha$, IL-6, and IL-10 levels showed no significant difference between the two groups (8).

The intestinal flora is a large group of microbial communities in the human intestine, which forms the intestinal mucosal barrier controlling nutrient absorption and metabolism, and is associated with systemic hypoinflammation and immune competence of the organism.
The results of a previous study showed that T2DM patients suffered from dysbacteriosis of the intestine, with varying degrees of alteration in diversity and abundance (9). In addition, the metabolites of T2DM have changed, and the host intestinal mucosa was damaged with higher permeability. Intestinal bacteria and their metabolites can cross the barrier and become ectopic, causing tissue inflammation and reducing the sensitivity of tissues to insulin, which leads to the development of T2DM $(10,11)$.

Some studies have shown that the intestinal flora and its metabolites can regulate the level of cytokines by affecting the immune response. However, the mechanism by which the intestinal flora induces T2DM through inflammation is not fully understood. Thus, a case-control experiment was implemented to determine whether cytokines play a role in reshaping intestinal flora as part of the development of T2DM and its mechanism. The results may provide a theoretical basis for diagnosing and treating T2DM more accurately and effectively.

We present the following article in accordance with the STROBE reporting checklist (available at https://dx.doi. org/10.21037/apm-21-2318).

\section{Methods}

\section{Research design and determination for research objects}

A case-control experiment was performed involving 29 patients (15 women and 14 men) who were initially diagnosed with T2DM at the clinical endocrinology clinic of the Affiliated Hospital of Chengdu University of Traditional Chinese Medicine from March 2020 to September 2020. A further 28 healthy individuals (16 women and $12 \mathrm{men}$ ) from the physical examination center of the institution were included as controls. All patients had not been previously treated with T2DM, and had no other metabolic or infectious disease, serious heart disease, liver disease, kidney injury, or intestinal disease, and had not taken antidiarrheal agents, probiotics, antibiotics, or other health care products within 3 months before participating in the current study.

All procedures performed in this study involving human participants were in accordance with the Declaration of Helsinki (as revised in 2013). This study was approved by the Medical Ethics Committee of the Affiliated Hospital of Chengdu University of Traditional Chinese Medicine (2020KL-031) and all participants signed informed consent. 


\section{Quantitative determination of plasma cytokines by flow cytometry}

The levels of interleukin-2 (IL-2), interleukin-4 (IL4), IL-10, TNF- $\alpha$, IFN- $\gamma$, and interleukin-17 (IL-17) in all plasma samples were measured quantitatively by BD ${ }^{\mathrm{TM}}$ CBA Human Th1/Th2 Cytokine Kit II (BD, USA, catalog number 551809), based on a method of capturing the soluble analyte with the known-sized fluorescent microspheres and the soluble analyte could be detected by flow cytometry. We used the classical gating strategy which is based on the optical signals, forward scattered light (FSC) versus side scattered light (SSC) parameters, collected by the cytometer, and secondly by propidium iodide (PI) staining (DNA intercalant with passive diffusion across cell membrane). This gating strategy of flow cytometry permits the exclusion of smaller elements such as cellular debris and any type of aggregates (12).

Each captured microsphere in the BD CBA kit (USA) had been conjugated with a specific antibody. The detection reagent was a mixture of phycoerythrin (PE)-coupled antibodies that provided a fluorescent signal proportional to the amount of analyte to be bound. When the capture microspheres and detection reagents were incubated with the analyte, a sandwich complex was formed, and the microsphere particles with fluorescence characteristics were measured and identified by BD FACS Canto II flow cytometer (USA). The PE fluorescence intensity of each sandwich complex revealed the corresponding cytokine concentration. Finally, the data were analyzed by FCAP array software, and the concentration of each cytokine was calculated from the standard curve.

\section{Fecal DNA extraction and $16 S$ rRNA gene sequencing}

Microbial DNA was isolated from each $250 \mathrm{mg}$ homogeneous fecal sample based on the instructions of the PowerSoil ${ }^{\circledR}$ DNA Isolation Kit (MoBio, USA), and the isolated DNA was stored at -20 to $-80{ }^{\circ} \mathrm{C}$ for further study. The target region of $16 \mathrm{~S}$ rRNA gene in each fecal sample were amplified by the following primers.

* Forward primer 27F: 5'-AGRGTTTGATYN TGGCTCAG-3';

* Reverse preimer1492R: 5'-TASGgHTACC TTGTTASGACTT-3'.

The obtained amplicons $(1,200-1,650 \mathrm{bp})$ were detected by gel electrophoresis. The purified PCR amplification product was sequenced by Pacbio SMRT with the carrier of SMRT chip sequencing. After corrected and filtered the original sequence of $\mathrm{PacBio}$, an effective sequence with a length of $1-1.8 \mathrm{~kb}$ was obtained, and after identified and removed the chimera, the Optimization-Circular Consensus Sequencing (Optimization-CCS) was obtained for subsequent analysis.

The above Optimization-CCS sequences were clustered by operational taxonomic units (OTUs) at $97 \%$ similarity level. Species annotations and abundance analysis were performed to reveal the species composition of the samples. The abundance and diversity of species were studied within individual samples by Alpha diversity analysis, which will count ACE, Chao1, Shannon, and Simpson indexes of each fecal sample, draw dilution and rank abundance curves. The differences in community composition and structure among samples were compared by Beta diversity analysis. Finally, the significance of the difference in genus level between the two groups was compared by Metastats Analysis and Principal Component Analysis (PCA) (the screening criteria of biomarker: LDA score $>4$ ).

\section{Statistical analysis}

All statistical analyses were performed by SPSS v23.0. Continuous variables are expressed as mean \pm standard deviation. Data with a normal distribution applied to $t$-test, otherwise, the non-parametric test was applied. The casecontrol group were compared by Mann-Whitney U test. The intestinal flora data of two independent samples were compared by the Mann-Whitney $\mathrm{U}$ test, with $\mathrm{P}<0.05$ considered as a statistically significant difference. The correlation and association analysis between the intestinal flora and cytokines were conducted by redundancy analysis (RDA) or canonical correspondence analysis (CCA). The strength of the linear relationship between the differential intestinal flora and cytokines were analyzed by Pearson correlation analysis technique.

\section{Results}

\section{Clinical and biochemical characteristics}

According to the inclusion and exclusion criteria, 29 patients initially diagnosed T2DM as cases and 28 healthy controls were included. The characteristics of these two groups were shown in Table 1. There was no statistical difference between the two groups in terms of gender and age $(\mathrm{P}>0.05)$. The renal parameters such as urea and 
Table 1 Clinical parameters of T2DM patients and healthy controls

\begin{tabular}{lccc}
\hline Clinical parameters & Control, $\mathrm{n}=28$ & T2DM, $\mathrm{n}=29$ & $\mathrm{P}$ \\
\hline Gender (female/male) & $16 / 12$ & $15 / 14$ & 0.681 \\
Age (years) & $46.71 \pm 2.17$ & $48.28 \pm 1.80$ & 0.581 \\
BMI $\left(\mathrm{kg} / \mathrm{m}^{2}\right)$ & $22.04 \pm 0.37$ & $24.72 \pm 0.55$ & 0.000 \\
FPG $(\mathrm{mg} / \mathrm{dL})$ & $5.14 \pm 0.10$ & $7.53 \pm 0.49$ & 0.000 \\
HbA1c $(\%)$ & $5.41 \pm 0.07$ & $7.13 \pm 0.35$ & 0.000 \\
TC $(\mathrm{mg} / \mathrm{dL})$ & $4.72 \pm 0.13$ & $4.76 \pm 0.13$ & 0.829 \\
TG $(\mathrm{mg} / \mathrm{dL})$ & $1.29 \pm 0.14$ & $1.80 \pm 0.18$ & 0.035 \\
HDL $(\mathrm{mg} / \mathrm{dL})$ & $1.51 \pm 0.08$ & $1.14 \pm 0.05$ & 0.000 \\
LDL $(\mathrm{mg} / \mathrm{dL})$ & $2.74 \pm 0.10$ & $3.10 \pm 0.12$ & 0.026 \\
Urea $(\mathrm{mmol} / \mathrm{L})$ & $4.60 \pm 0.39$ & $5.30 \pm 0.31$ & 0.162 \\
Crea $(\mu \mathrm{mol} / \mathrm{L})$ & $62.00 \pm 2.32$ & $60.69 \pm 2.21$ & 0.660 \\
FINS $(\mu \mathrm{U} / \mathrm{mL})$ & $8.42 \pm 0.658$ & $11.43 \pm 1.16$ & 0.030 \\
\hline
\end{tabular}

All parameters are mean \pm SD. BMI, body mass index; FPG, fasting plasma glucose; HbA1c, hemoglobin A1c; TC, total cholesterol; TG, triglyceride; HDL, high-density lipoprotein; LDL, low-density lipoprotein; FINS, fasting serum insulin.

creatinine were all within the normal range. While HDL was decreased $(\mathrm{P}<0.001)$ in patients with $\mathrm{T} 2 \mathrm{DM}$, the levels of FPG $(\mathrm{P}<0.001)$, HbA1c $(\mathrm{P}<0.001)$, FINS $(\mathrm{P}=0.03)$, body mass index $\left(B M I=\frac{\text { Weight }(\mathrm{kg})}{\operatorname{Height}(\mathrm{m})^{2}}\right)(\mathrm{P}<0.001), \mathrm{TG}(\mathrm{P}=0.035)$, and LDL $(\mathrm{P}=0.026)$ were significantly increased, indicating T2DM patients may potentially be at risk of cardiovascular disease.

\section{Plasma cytokine expression}

The plasma cytokine including IL-2, IL-4, IL-6, IL10 , TNF- $\alpha$, IFN- $\gamma$, and IL-17 were tested both in T2DM patients and healthy controls. Figure 1 shows that the concentration of the pro-inflammatory cytokines, including IL-2 ( $\mathrm{P}=0.0000006)$, IL-6 $(\mathrm{P}=0.000193)$, TNF- $\alpha$ $(\mathrm{P}=0.016), \mathrm{IFN}-\gamma(\mathrm{P}=0.000036)$, and $\mathrm{IL}-17(\mathrm{P}=0.004)$ in $\mathrm{T} 2 \mathrm{DM}$ patients were significantly higher than those of controls. The concentration of IL-4 ( $\mathrm{P}=0.000010)$, an antiinflammatory cytokine, was also significantly higher in T2DM patients than that in the healthy control group, and IL-10 $(\mathrm{P}=0.053)$ demonstrated a rising trend in T2DM patients. These results indicated that patients initially diagnosed as T2DM are in a state of inflammation. Studies have shown that hyperglycemia can cause oxidative stress on T-lymphocytes by activating reactive oxygen species and protein kinase $\mathrm{C}$, which promote the secretion of cytokines and progression of inflammation causing damage to cellular immune function (13).

\section{Abundance, diversity, and different composition of intestinal flora}

Differences between the Shannon index $(2.58 \pm 0.09$ vs. 2.16 $\pm 0.12, \mathrm{P}<0.05)$, Chao 1 index $(94.34 \pm 4.15$ vs. $79.06 \pm 5.56, \mathrm{P}<0.05)$, and ace index $(94.36 \pm 3.99$ vs. $79.56 \pm 5.58, \mathrm{P}<0.05)$ of healthy individuals and T2DM patients were statistically significant, while the Simpson index $(0.18 \pm 0.018$ vs. $0.25 \pm 0.028, \mathrm{P}>0.05)$ has no statistical significance but with an increasing trend in value (Figure 2A). These results showed that the abundance and diversity of bacteria in T2DM patients were reduced compared with healthy controls. Furthermore, PCA analysis indicated that the composition of the intestinal flora of T2DM patients had some specific changes compared with healthy controls (Figure 2B).

The composition of the intestinal flora was assessed by comparing the relative proportions of dominant taxonomic units at the phylum level. As shown in Figure 2C, compared to healthy controls, the abundance of Firmicutes and Proteobacteria in T2DM patients was increased, whereas that of Bacteroides was decreased, and the ratio of Firmicutes/Bacteroidetes was significantly increased. 16 differentially expressed biomarkers (Figure 2D) were found from LDA distribution histograms which were generated by LEfSe analysis. At the species level, compared to healthy controls, the abundance of Bacteroides stercoris $(\mathrm{P}=0.0068)$, Bacteroides_uniformis $(\mathrm{P}=0.033)$, and Phascolarctobacterium_faecium $(\mathrm{P}=0.033)$ were lower in patients with T2DM, while Megamonas_funiformis $(\mathrm{P}=0.0016)$ and Escherichia $(\mathrm{P}=0.049)$ were enriched (LAD score $\log 10>4.0)$. These above results revealed the difference in the composition of the intestinal microbiota between the two groups, showing the unique diversity and distribution characteristics of the intestinal microbiota in patients initially diagnosed as T2DM.

\section{Correlation analysis of intestinal flora and cytokines}

The RDA/CCA correlation analysis chart shows the association of the intestinal flora and cytokines in diabetic patients (Figure 3A). The 12 categories of intestinal bacteria 


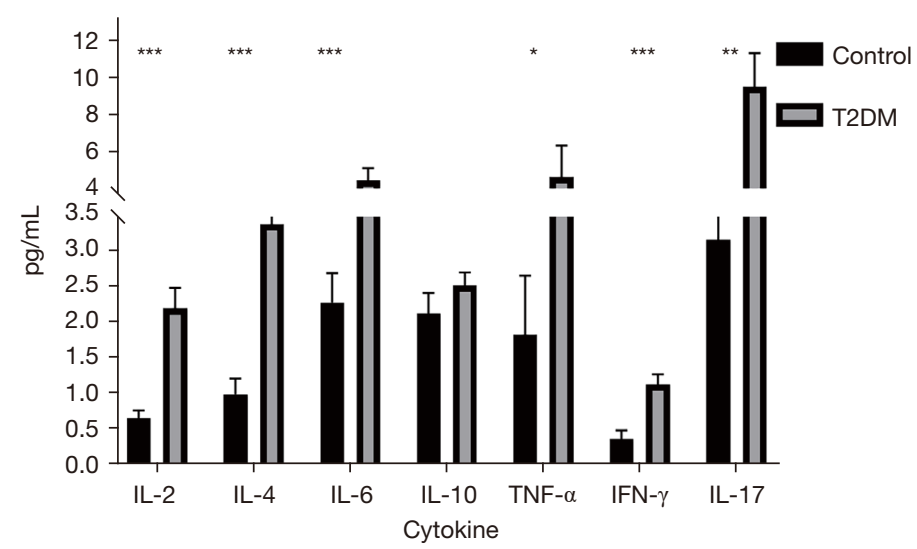

Figure 1 Cytokine level of T2DM patients and healthy controls. All parameters mean $\pm \mathrm{SD}$ is reported. *, $\mathrm{P}<0.05 ;{ }^{* *}, \mathrm{P}<0.01 ;{ }^{* * *}, \mathrm{P}<0.001$. T2DM, type 2 diabetes mellitus; IL-2, interleukin-2; IL-4, interleukin-4; IL-10, interleukin-10; TNF- $\alpha$, tumor necrosis factor $\alpha$; IFN- $\gamma$, interferon- $\gamma$; IL-17, interleukin-17.

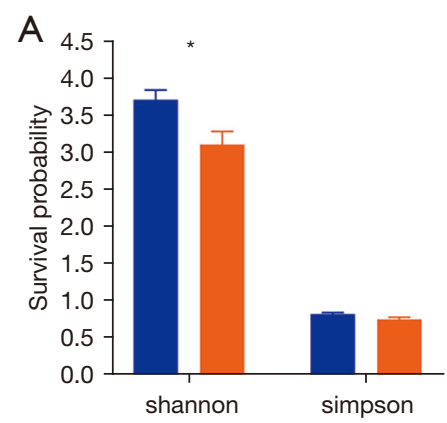

C

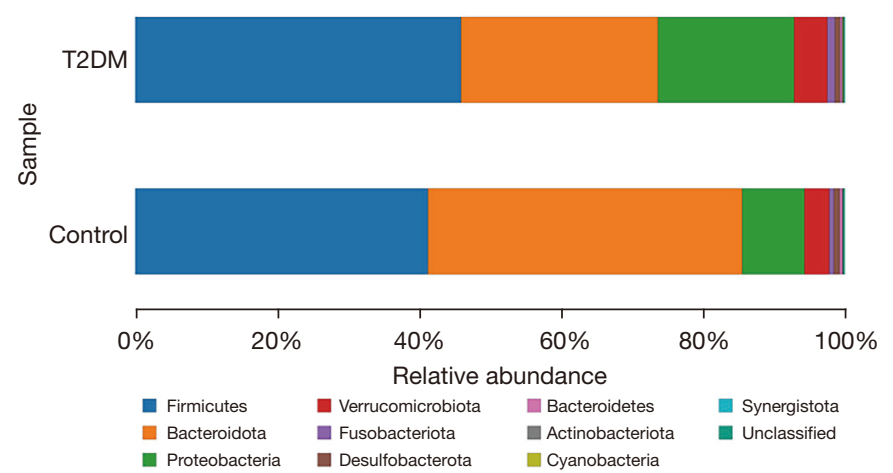

B
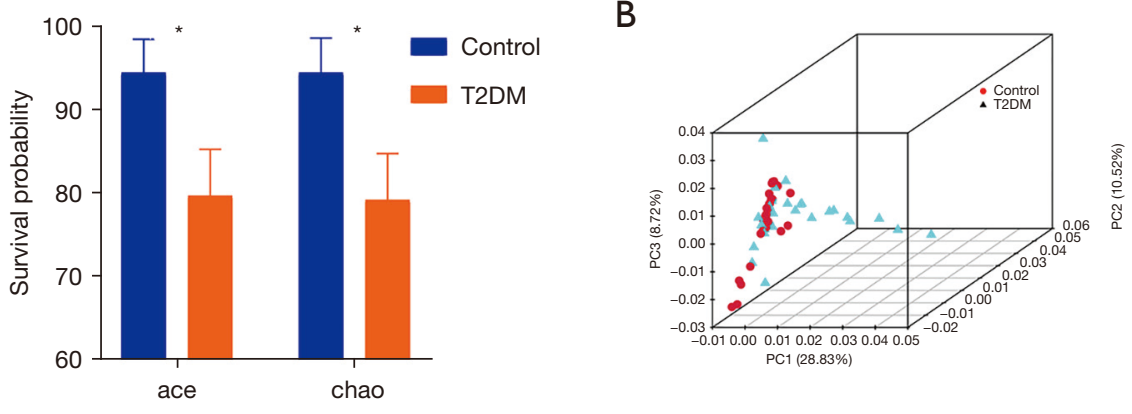

D

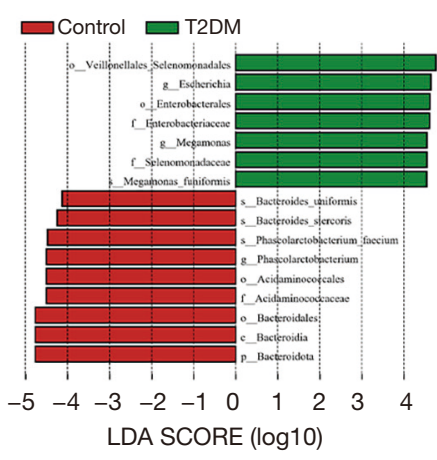

Figure 2 Abundance, diversity, and different composition of intestinal flora. (A) Species diversity differences between T2DM patients and healthy controls were estimated by the Shannon, Simpson, ace, and Chao1 indexes. The abundance of species was measured by Chaol and ACE indexes, to indicate the specific types of species. The diversity of species was measured by Shannon and Simpson indexes, which was affected by species abundance and community evenness in sample communities. *, $\mathrm{P}<0.05$; (B) the bacterial structural clustering is shown in a PCA map based on relative abundance OTUs (97\% similarity level); (C) relative abundance of the top 15 abundant bacterial phylum in two groups; (D) the distribution histogram of LDA value. T2DM, type 2 diabetes mellitus; OTU, operational taxonomic unit. 

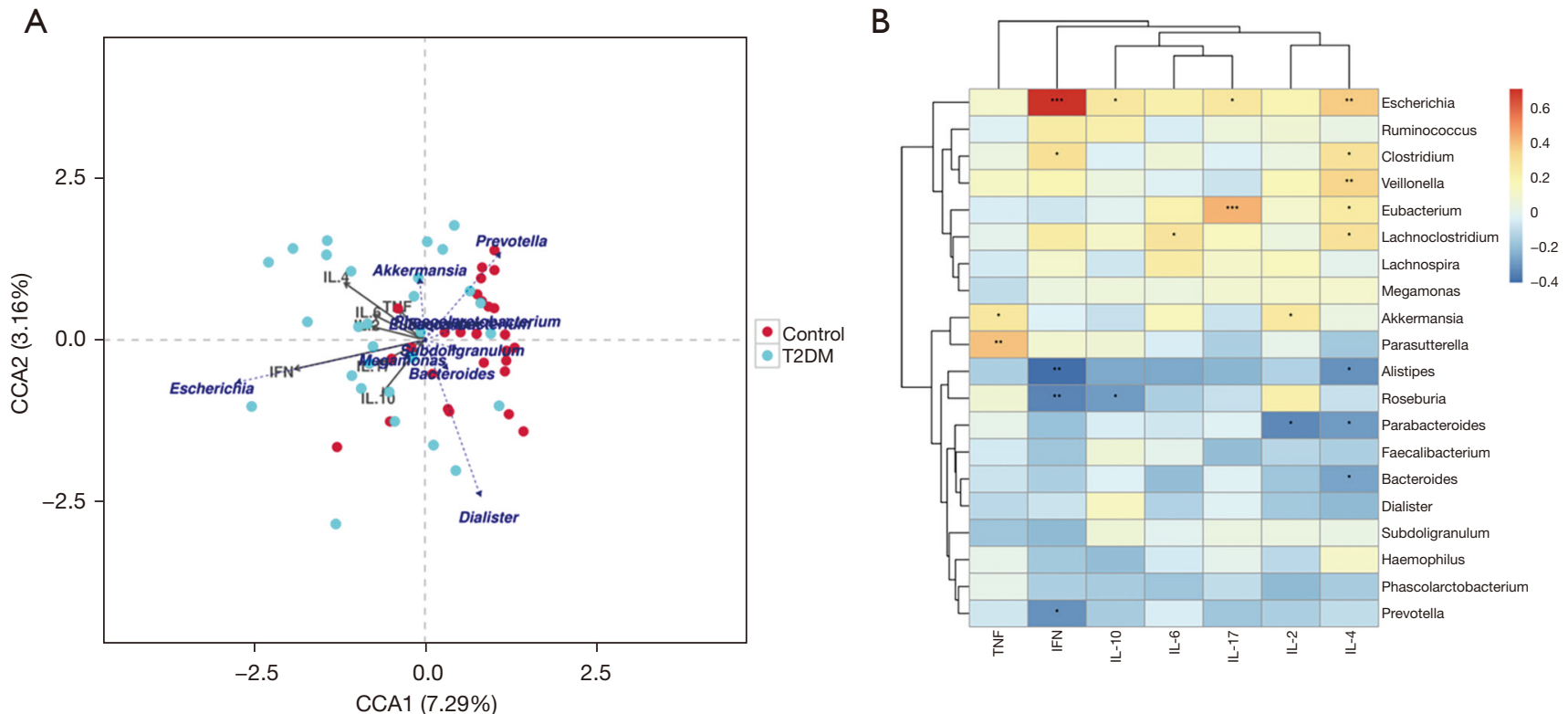

Figure 3 Correlation analysis of intestinal flora and cytokines. (A) RDA/CCA analysis of cytokines and intestinal flora. (B) Pearson correlation analysis of cytokines and intestinal flora. RDA, redundancy analysis; CCA, canonical correspondence analysis. *, $0.2<|\mathrm{r}|<0.4$; **, $0.4<|r|<0.6 ; * * *,|r|>0.6$.

in the top 20 in abundance have different degrees of positive and negative correlation with cytokines at the genus level (Figure 3B) by Pearson correlation analysis and visualization. Among them, the differentially expressed Escherichia had the most significant positive correlation with IFN- $\gamma(\mathrm{r}=0.73$, $\mathrm{P}<0.001$ ), while no cytokines had significant negative correlation with Escherichia.

\section{Discussion}

In the current study, we evaluated the differences in cytokine levels and the diversity and richness of intestinal flora of healthy controls and patients with T2DM. Compared to healthy controls, the level of FPG, TG, FINS, BMI, and other basic clinical indicators were remarkably increased with statistical significance in T2DM patients, and with an increasing trend of TC and LDL. Related studies have found that elevated TG levels were the most common manifestation of metabolic disorders in T2DM patients (14). TG is the major source of energy in the body. It will be stored in adipose tissue and induce inflammation of adipose tissue when it is in excess. In addition, we found that the serum levels of IL2 , IL-6, TNF- $\alpha$, IFN- $\gamma$, and IL-17 in T2DM patients were significantly higher than those in the control group, suggesting patients with T2DM may have an inflammatory state, which was consistent with other studies (15). The activation and accumulation of M1 macrophages participate in the occurrence of T2DM through the levels of IFN- $\gamma$ or lipopolysaccharide (LPS), the subsequently secreted IL6 , IL-1 $\beta$, TNF- $\alpha$, and other pro-inflammatory cytokines which can induce chronic low-level inflammation and metabolic damage $(16,17)$.

Our results also showed that patients with T2DM suffered from intestinal flora disorders, with reduced species diversity and altered abundance of dominant species. The changes of abundance, including Firmicutes and Proteobacteria increased, Bacteroidetes significantly decreased, and the ratio of Bacteroidetes/Firmicutes significantly decreased, which were consistent with earlier studies $(18,19)$. The decrease in Bacteroidetes and increase in Firmicutes may be related to some enzymes that can make polysaccharides break down and enhance the ability to obtain energy from food, which could cause mild systemic inflammation $(20,21)$. These findings indicated that an extremely complex network of regulatory mechanisms stemming from intestinal flora imbalance which affects the occurrence and development of T2DM may exist.

Furthermore, we found that Megamonas_funiformis and Escherichia were significantly enriched in the intestines of 
T2DM patients at the genus level. The linear relationship between cytokine levels and intestinal flora was analyzed by Pearson correlation, revealing a positive correlation between Escherichia and IFN- $\gamma(\mathrm{R}=0.73, \mathrm{P}<0.001)$, and suggesting Escherichia may be involved in the development of T2DM in mutual regulation with IFN- $\gamma$. As a pro-inflammatory cytokine produced by $\mathrm{NK}$ cells, IFN- $\gamma$ plays a role as the Janus kinase/signal transducer and activator of tran-ions (JAK/STAT) (22). Previous experiments have shown that VAT was the major source of chronic inflammation and an important underlying cause of inducing insulin resistance and developing T2DM, while VAT inflammation seemed to be related to the accumulation of M1 macrophages for a pro-inflammatory M1 phenotype caused by the conversion of ATM from an anti-inflammatory M2 phenotype $(23,24)$. M1 macrophages are the most abundant immune cells infiltrating VAT, which is the key mediator of VAT inflammation (25), and the depletion of M1 macrophages in humans and mice has been shown to significantly improve systemic insulin resistance (26). Thus, macrophages are critical for the development of VAT inflammation, which seems to be the main source of IL- 6 , IL- $1 \beta$, and TNF- $\alpha$ pro-inflammatory cytokines contributing to insulin resistance (27), while IFN- $\gamma$ is a key factor that activates macrophages and induces their differentiation into the pro-inflammatory M1 phenotype (5). In the context of host obesity, the NK cell activating receptor 1 (NCR1) on adipocytes can be up-regulated, which stimulates NK cell proliferation and IFN- $\gamma$ production (28), and upregulates the JAK/STAT pathway. Studies have found that mice with IFN- $\gamma$ deficiency reduced the differentiation and accumulation of M1 macrophages in VAT, and the insulin sensitivity of high-fat diet feeding could be improved $(7,29)$. In addition, inhibition of the JAK/STAT signaling pathway from IFN- $\gamma$ subsequent pathway and the expression of suppressor of cytokine signaling-1 (SOCS1) could inhibit the effect of IFN- $\gamma$ on the differentiation and accumulation of macrophages towards M1 type (30,31). Therefore, IFN- $\gamma$ produced by NK cells may be an enabling factor that mediates the onset of VAT inflammation and induces insulin resistance in T2DM.

Previous studies have demonstrated that Escherichia abundance in the intestinal flora of patients with T2DM increases dynamically with disease progression (32). The pro-inflammatory nature of Escherichia which may be related to LPS, a component of cell wall, has been repeatedly reported. When the intestinal flora is dysregulated and intestinal permeability is increased, LPS can easily absorbed into the blood through the intestinal barrier and subsequently binds to Toll-like receptor 4 (TLR4) on the surface of macrophages. Together, these form a complex that upregulate NF- $\mathrm{KB}$ expression through a related pathway (33), which could promote the release of IL-6, TNF- $\alpha$, INF- $\gamma$, and other inflammatory factors in various tissues and induce insulin resistance $(34,35)$. TLR receptor is positively correlated with long-term blood glucose marker HbA1c (11), and activation of TLR4 may also induce synthesis of TG (36). Except for subclinical inflammation and insulin resistance, previous studies have shown that Escherichia could also increase the visceral fat content of mice. This is consistent with the performance of high-fat fed mice (37), which could up-regulate the NCR1 receptor on adipocytes, releases IFN- $\gamma$, promote the accumulation of M1-type macrophages, and exacerbate VAT inflammation-induced insulin resistance. Furthermore, Escherichia can inhibit the production of short-chain fatty acids (SCFA) which can induce the production of regulatory $\mathrm{T}$ cells (Tregs) outside the thymus. Tregs can inhibit the secretion of IFN- $\gamma$, reduce the activation of ATM, and improve insulin resistance in adipose tissue. Therefore, increases in Escherichia can reduce the production of SCFA, weakening the inhibitory effect of SCFA on VAT inflammation, further aggravating insulin resistance (15).

A paper published in Nature has evaluated the association of Vitamin D metabolites and the gut microbiome in older men (38). Their results showed that intestinal flora was correlated to vitamin D. Patients need to adjust their vitamin $\mathrm{D}$ levels to improve their intestinal flora. Thus, we suggested people with T2DM maintain vitamin D levels to improve the intestinal flora, including exposure to the sun, strengthen the sports, and eating more foods containing vitamin $\mathrm{D}$.

In conclusion, our results indicated that dysbiosis of the intestinal flora may lead to disruption of the dynamic balance of the host immune system-intestinal flora interaction network, and gradually lead to immune pathological processes such as systemic or local chronic inflammation, ultimately causing insulin hypersecretion and the development of T2DM. IFN- $\gamma$ and Escherichia were found to be significantly altered in T2DM patients, we speculate that the network of action between the two may have a role in driving VAT inflammation by blocking insulin signaling in cells and reducing insulin sensitivity, which may eventually lead to insulin resistance. Furthermore, the immune mechanism of T2DM induced by intestinal flora through chronic inflammatory pathways was discovered by 
exploring the correlation between differentially expressed intestinal microorganisms and the level of organismal cytokines, which may represent a breakthrough in the early diagnosis and treatment of T2DM. However, our observation about correlation of the intestinal bacteria (Escherichia) and the inflammatory factor IFN- $\gamma$ is only a preliminary finding, further in-depth exploration should be performed to determine the target genes that co-regulate intestinal flora and cytokines in the promotion of low-grade inflammation in T2DM. Moreover, the early diagnosis and precise treatment of T2DM can be realized by monitoring and regulating the expression of target genes.

\section{Acknowledgments}

Funding: Key R\&D Project of Sichuan Provincial Department of Science and Technology (2020YFS0375).

\section{Footnote}

Reporting Checklist: The authors have completed the STROBE guideline checklist. Available at https://dx.doi. org/10.21037/apm-21-2318

Data Sharing Statement: Available at https://dx.doi. org/10.21037/apm-21-2318

Conflicts of Interest: All authors have completed the ICMJE uniform disclosure form (available at https://dx.doi. org/10.21037/apm-21-2318). The authors have no conflicts of interest to declare.

Ethical Statement: The authors are accountable for all aspects of the work in ensuring that questions related to the accuracy or integrity of any part of the work are appropriately investigated and resolved. All procedures performed in this study involving human participants were in accordance with the Declaration of Helsinki (as revised in 2013). This study was approved by the Medical Ethics Committee of the Affiliated Hospital of Chengdu University of Traditional Chinese Medicine (2020KL-031) and all participants signed informed consent.

Open Access Statement: This is an Open Access article distributed in accordance with the Creative Commons Attribution-NonCommercial-NoDerivs 4.0 International
License (CC BY-NC-ND 4.0), which permits the noncommercial replication and distribution of the article with the strict proviso that no changes or edits are made and the original work is properly cited (including links to both the formal publication through the relevant DOI and the license). See: https://creativecommons.org/licenses/by-nc-nd/4.0/.

\section{References}

1. Li Y, Teng D, Shi X, et al. Prevalence of diabetes recorded in mainland China using 2018 diagnostic criteria from the American Diabetes Association: national cross sectional study. BMJ 2020;369:m997.

2. Yang Y, Chan L. Monogenic Diabetes: What It Teaches Us on the Common Forms of Type 1 and Type 2 Diabetes. Endocr Rev 2016;37:190-222.

3. Song S, Lee JE. Dietary Patterns Related to Triglyceride and High-Density Lipoprotein Cholesterol and the Incidence of Type 2 Diabetes in Korean Men and Women. Nutrients 2018;11:8.

4. Caslin HL, Bhanot M, Bolus WR, et al. Adipose tissue macrophages: Unique polarization and bioenergetics in obesity. Immunol Rev 2020;295:101-13.

5. Goldszmid RS, Caspar P, Rivollier A, et al. NK cellderived interferon- $\gamma$ orchestrates cellular dynamics and the differentiation of monocytes into dendritic cells at the site of infection. Immunity 2012;36:1047-59.

6. Han MS, Jung DY, Morel C, et al. JNK expression by macrophages promotes obesity-induced insulin resistance and inflammation. Science 2013;339:218-22.

7. O'Rourke RW, White AE, Metcalf MD, et al. Systemic inflammation and insulin sensitivity in obese IFN- $\gamma$ knockout mice. Metabolism 2012;61:1152-61.

8. Kartika R, Purnamasari D, Pradipta S, et al. Impact of Low Interferon- $\gamma$ and IL-10 Levels on TNF- $\alpha$ and IL-6 Production by PHA-Induced PBMCs in Type 2 Diabetes Mellitus. J Inflamm Res. 2020;13:187-93.

9. Miyamoto J, Kasubuchi M, Nakajima A, et al. The role of short-chain fatty acid on blood pressure regulation. Curr Opin Nephrol Hypertens 2016;25:379-83.

10. Klüppelholz B, Thorand B, Koenig W, et al. Association of subclinical inflammation with deterioration of glycaemia before the diagnosis of type 2 diabetes: the KORA S4/F4 study. Diabetologia 2015;58:2269-77.

11. Bagarolli RA, Tobar N, Oliveira AG, et al. Probiotics modulate gut microbiota and improve insulin sensitivity in 
DIO mice. J Nutr Biochem 2017;50:16-25.

12. Sabido O, Figarol A, Klein JP, et al. Quantitative Flow Cytometric Evaluation of Oxidative Stress and Mitochondrial Impairment in RAW 264.7 Macrophages after Exposure to Pristine, Acid Functionalized, or Annealed Carbon Nanotubes. Nanomaterials (Basel) 2020;10:319.

13. Thaiss CA, Levy M, Grosheva I, et al. Hyperglycemia drives intestinal barrier dysfunction and risk for enteric infection. Science 2018;359:1376-83.

14. Zhao J, Zhang Y, Wei F, et al. Triglyceride is an independent predictor of type 2 diabetes among middleaged and older adults: a prospective study with 8-year follow-ups in two cohorts. J Transl Med 2019;17:403.

15. Saad MJ, Santos A, Prada PO. Linking Gut Microbiota and Inflammation to Obesity and Insulin Resistance. Physiology (Bethesda) 2016;31:283-93.

16. McLaughlin T, Ackerman SE, Shen L, et al. Role of innate and adaptive immunity in obesity-associated metabolic disease. J Clin Invest 2017;127:5-13.

17. Wensveen FM, Valentić S, Šestan M, et al. The "Big Bang" in obese fat: Events initiating obesity-induced adipose tissue inflammation. Eur J Immunol 2015;45:2446-56.

18. Cho I, Yamanishi S, Cox L, et al. Antibiotics in early life alter the murine colonic microbiome and adiposity. Nature 2012;488:621-6.

19. Turnbaugh PJ, Bäckhed F, Fulton L, et al. Diet-induced obesity is linked to marked but reversible alterations in the mouse distal gut microbiome. Cell Host Microbe 2008;3:213-23.

20. Turnbaugh PJ, Ley RE, Mahowald MA, et al. An obesityassociated gut microbiome with increased capacity for energy harvest. Nature 2006;444:1027-31.

21. Ley RE, Bäckhed F, Turnbaugh P, et al. Obesity alters gut microbial ecology. Proc Natl Acad Sci U S A 2005;102:11070-5.

22. McGillicuddy FC, Chiquoine EH, Hinkle CC, et al. Interferon gamma attenuates insulin signaling, lipid storage, and differentiation in human adipocytes via activation of the JAK/STAT pathway. J Biol Chem 2009;284:31936-44.

23. Lumeng CN, Bodzin JL, Saltiel AR. Obesity induces a phenotypic switch in adipose tissue macrophage polarization. J Clin Invest 2007;117:175-84.

24. Patsouris D, Li PP, Thapar D, et al. Ablation of CD11cpositive cells normalizes insulin sensitivity in obese insulin resistant animals. Cell Metab 2008;8:301-9.

25. Odegaard JI, Chawla A. Alternative macrophage activation and metabolism. Annu Rev Pathol 2011;6:275-97.

26. Koppaka S, Kehlenbrink S, Carey M, et al. Reduced adipose tissue macrophage content is associated with improved insulin sensitivity in thiazolidinedione-treated diabetic humans. Diabetes 2013;62:1843-54.

27. Starr ME, Evers BM, Saito H. Age-associated increase in cytokine production during systemic inflammation: adipose tissue as a major source of IL-6. J Gerontol A Biol Sci Med Sci 2009;64:723-30.

28. Wensveen FM, Jelenčić V, Valentić S, et al. NK cells link obesity-induced adipose stress to inflammation and insulin resistance. Nat Immunol 2015;16:376-85.

29. Wong N, Fam BC, Cempako GR, et al. Deficiency in interferon-gamma results in reduced body weight and better glucose tolerance in mice. Endocrinology 2011;152:3690-9.

30. Liang YB, Tang H, Chen ZB, et al. Downregulated SOCS1 expression activates the JAK1/STAT1 pathway and promotes polarization of macrophages into M1 type. Mol Med Rep 2017;16:6405-11.

31. Ghazarian M, Revelo XS, Nøhr MK, et al. Type I Interferon Responses Drive Intrahepatic T cells to Promote Metabolic Syndrome. Sci Immunol 2017;2:eaai7616.

32. Gao R, Zhu C, Li H, et al. Dysbiosis Signatures of Gut Microbiota Along the Sequence from Healthy, Young Patients to Those with Overweight and Obesity. Obesity (Silver Spring) 2018;26:351-61.

33. Wang G, Hu Z, Fu Q, et al. Resveratrol mitigates lipopolysaccharide-mediated acute inflammation in rats by inhibiting the TLR4/NF- Bp65/MAPKs signaling cascade. Sci Rep 2017;7:45006.

34. Domingueti CP, Dusse LM, Carvalho Md, et al. Diabetes mellitus: The linkage between oxidative stress, inflammation, hypercoagulability and vascular complications. J Diabetes Complications 2016;30:738-45.

35. Cani PD, Bibiloni R, Knauf C, et al. Changes in gut microbiota control metabolic endotoxemia-induced inflammation in high-fat diet-induced obesity and diabetes in mice. Diabetes 2008;57:1470-81.

36. Huang YL, Morales-Rosado J, Ray J, et al. Toll-like receptor agonists promote prolonged triglyceride storage in macrophages. J Biol Chem 2014;289:3001-12.

37. Sun Q, Zhang S, Liu X, et al. Effects of a probiotic 
intervention on Escherichia coli and high-fat dietinduced intestinal microbiota imbalance. Appl Microbiol Biotechnol 2020;104:1243-57.

38. Thomas RL, Jiang L, Adams JS, et al. Vitamin D metabolites and the gut microbiome in older men. Nat Commun 2020;11:5997.

(English Language Editor: B. Draper)

Cite this article as: Lv M, Li L, Li W, Yang F, Hu Q, Xiong D. Mechanism research on the interaction regulation of Escherichia and IFN- $\gamma$ for the occurrence of T2DM. Ann Palliat Med 2021;10(10):10391-10400. doi: 10.21037/apm-21-2318 